\title{
Mobility Impact on MANET Routing Protocols In Grid Networks
}

\author{
Zeinab R. Khaleel,and Mahmoud M.AL-Quzwini \\ College of Engineering, Alnahrain University, \\ Baghdad, Iraq \\ Department of Computer Engineering
}

\begin{abstract}
Mobile Ad-Hoc Networks (MANETS) is a collection of wireless mobile nodes that are able to dynamically form a temporary network without any aid from fixed infrastructure or centralized administration due to no wired backbone.Ad Hoc networks are formed spontaneously and the nodes are highly mobile.Thispaper presents performance evaluations, comparisons, andanalysis for three routing protocols (AODV, DSR, and OLSR) to bring out their relative meritsundervarying network size and mobilitywith various speed and pause times. The simulation is carried out using OMNET++ simulator based on the quantitative basic parameters like throughput, Packet transmission Ratio (PTR),packet transmission time delay and protocol overhead. The nodes are distributed randomly in a grid network topology and mobile nodes moving using Random Waypoint mobility models. The results demonstrate that, undervarious node speeds and pause timesfor different network size, AODVoutperforms DSR and OLSR protocols with respect to networkthroughput (by $0.9 \%$ and $4.4 \%$, respectively). For protocoloverhead,DSR is about $13.4 \%$ and $65.5 \%$ below AODV and OLSR protocols respectively. In contrast, thepacket transmission time delay when using OLSR is shorter than whenusing both AODV (by $81.7 \%$ ) and DSR (by $76.7 \%$ ).
\end{abstract}

Keywords --MANET; AODV; DSR; OLSR.

\section{Council for Innovative Research}

Peer Review Research Publishing System

Journal: INTERNATIONAL JOURNAL OF COMPUTERS \& TECHNOLOGY

Vol 11, No. 6

editor@cirworld.com

www.cirworld.com, member.cirworld.com 


\section{INTRODUCTION}

Mobile Ad Hoc network (MANET) is the most innovative and challenging area of wireless networking. MANET is an autonomous collection of mobile users that seamlesslycommunicate based onradio to radio multi-hoping over relatively band width constrained wireless links withoutany preexisting communication architecture [1-3].

With changing technology mobile devices getting smaller, cheaper, more convenient, and more powerful, they also run more applications and network services [4]. MANET is mobility where all nodes are allowed to move in different a dimension which results in dynamic topology that leads to link breakage and path loss [5]. A major challenge that lies in MANET is route selection due to the limited resources and transmission range for a wireless radio, which is typically limited to $200-300 \mathrm{~m}$ [6-8]. Therefore, therouting protocol plays a very important role that affects the network's performance [7]. Different types of routing protocols give different network performances. These differences arise due to the differentmechanisms of these protocols. we needed to use a routing protocol that had very low overhead yet was able to react quickly to changes in the network, providing highly reactive service to help ensure successful delivery of data packets in spite of node movement or other changes in network conditions [1, 6, 9].

The main contributions of this paper are to evaluate, analyze, and compare the performance of three types ofrouting protocols: Ad Hoc on Demand Distance Vector routing protocol (AODV), Dynamic Source Routingprotocol (DSR), and Optimized Link State Routing protocol (OLSR), both DSR, AODV are on demand protocols. WhileOLSR is a proactive routing protocol.The motivation behind this comparison is to understand their internal working mechanism and bring out situations where one is preferred than the other in order to find how to improve the routing performance $[1,5]$.

The rest of the paper is organized as follows.Section II givesan overview of the related work. Section III explains routing protocols in MANET, in SectionsIV, V, andVI, DSR, AODV and OLSR routing protocols mechanismsare explained in details respectively. In Section VII, Random Waypoint Mobility Model isstated. Protocols Performance Metrics are illustrated in section VIII. Section XI discusses our Simulation Scenario and parameters setup, while the experimental results of these simulations are discussed in section X. Our conclusions and future work are listed in Section XI.

\section{RELATED WORK}

Several performance evaluations of MANET routing protocols have been done in the literature:

Divecha et al., in 2007[10] studied the effects of various mobility models on the performance of two routing protocols Dynamic Source Routing (DSR-Reactive Protocol) and Destination-Sequenced Distance-Vector (DSDV-Proactive Protocol) by considering four mobility scenarios: Random Waypoint, Group Mobility, Freeway and Manhattan models. Performance comparison has also been conducted across varying node densities and number of hops. Experiment results illustrate that performance of the routing protocol varies across different mobility models, node densities and length of data paths.

Gowrishankar et al., in 2007[11]made a performance comparison of two prominent routing protocols in wireless Ad Hoc networks: the Ad Hoc On-Demand Distance Vector Routing (AODV) and the Optimized Link State Routing (OLSR) protocol. The performance differentials were analyzed using various metrics. The comparison showed that the OLSR protocol is more efficient in networks with high density and highly sporadic traffic. Moreover, this comparison illustrates that the AODV protocol will perform better in the networks with static traffic, when the number of source and destination pairs is relatively small for each host.

Kumar et al., in 2009[5] analyzed the performance of AODV and DSR protocols by varying network load, mobility and type of traffic (CBR, TCP) using NS2. Both DSR and AODV share similar on demand behavior, but the protocols internal mechanism leads to significant performance differences.

Hu et al., in 2010[12] studied the impacts of mobility on routing protocol performance of MANETs with simulation, which uses ns-2 as the simulation tool, 802.11 as the wireless MAC protocol and AODV as the routing protocol. The results show that the increase in mobility does not reduce the routing protocol performance distinctly. In some circumstances, it even increases the routing protocol performance.

Amnai et al., in 2011[13] studied the impact of three random mobility models (Random Waypoint, Random Direction and Mobgen-Steady State) on the performance of On-Demand Distance Vector Routing (AODV) protocol. In this thesis the impact of node density with two different values of pause time for a fixed speed was examined on performance (Endto-End Delay, Throughput and Packet Delivery Ratio). Experimental results illustrate that the performance of the routing protocol varies across different mobility models.

Alshowkanet al., in 2012[14] evaluated Dynamic MANET on-demand routing protocol (DYMO), Ad Hoc On-Demand Distance Vector Routing protocol (AODV) and Dynamic Source Routing protocol (DSR). The evaluation of performance is based on throughput, dropped packets and end-to-end delay.

\section{ROUTING PROTOCOLS IN MANETS}

Researcher Community has broadly classifiedthe routing protocols into two categories. These are Proactive (Table Driven) routingprotocols, because the routing table of node is periodically exchanged and updated.An example of proactive routing protocol is the Optimized Link State Routing Protocol (OLSR) [6, 4].A reactive protocol on the other handfinds the route only to needed nodes only ondemand.In reactive or on demand protocols the up-todate routing table is not retained if there is no communication. It will discover the route only when it is having the data tosend. Examples of 
reactive routing protocols are the Dynamic Source Routing (DSR) and Ad Hoc On-demand Distance Vector routing (AODV) [6].

In order toget the advantages of both table driven and on- demand routing protocols, one can combine theapproaches to form a hybrid protocol. Various protocols have been developed under thesecategories over the years. Zone Routing Protocol (ZRP) offers a hybrid protocol [9].

The next sub-section describes the basic features of these protocols. For a more detailed description, the reader is referred to the respective RFCs [15-17].

\section{DYNAMIC SOURCE ROUTING (DSR)}

The Dynamic Source Routing (DSR) protocol is a simple and efficient routing protocol designed specifically for usein multi-hop wirelessAdHoc networks of mobile nodes [6]. Also it is a reactive, on-demandrouting protocol based on a method known as source routing, a routing technique in which the sender of the packet determines the complete sequence of the nodes through which to forward the packet. The sender explicitly lists this route in the packet's header, identifying each forwarding "hop" by the address of the next node to which to transmit the packet on its way to the destination host [1].

Nodes in DSR 'learn' and cache multiple routes to each destination (either as a response to a request, forwarding, or overhearing) to be used in case of route loss. In addition, this also helps in reducing routing overheads. The on-demand feature of DSRreduces the bandwidth use, especially in cases where the mobility is low.

The DSR protocol consists of two mechanisms:

Route Discovery and Route Maintenance, which work together to enable nodes to discover routes to destinations, and to maintain the routes to prevent any loss[6, 10].

\section{Route Discovery Mechanism in DSR}

When a source node $S$ wants to send message to the destination node $D$, as shown in Figure 1 , it checks its routing table. If no route is found to the destination, then node " $S$ " activates the route discovery procedure to find the route to the destination, node " $D$ " by broadcasting the RREQ packet to its neighbors ( $A, E, F)$. The intermediate nodes (A, $E, F)$ on receive the RREQ packet rebroadcast the packet to its neighbors until the request reaches the requested node "D". Any node participating in route discovery can learn routes from passing packets and gather this routing information into its route cache. When the destination node $D$ receives two or more RREQ packets from the same source through different routes, it checks its cached routes tofind the two best routes based on the numberof hopes. The route which has least number of hops it becomes primary $<\mathrm{S}, \mathrm{F}, \mathrm{G}>$. Otherwise it uses the reverse of the route that has been used by the request message to send the reply $[6,18]$.

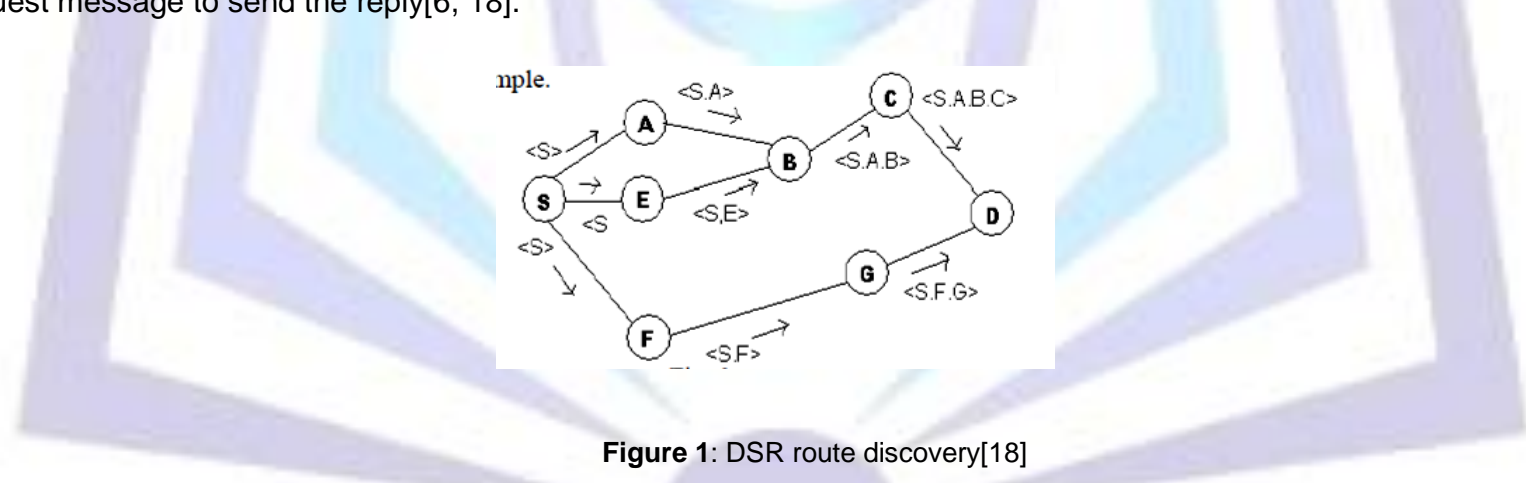

\section{Route Maintenance Mechanism in DSR}

Route Maintenance is used to detect if the network topology has changed such that the link used by this packet is broken, and has to inform the source about the route breakage by sending a route error message (RERR). When the source node is informed about the route breakage, it removes the broken route from its cache and starts repairing the route to the destination by looking for another route in its cached routes. If no route is found tothe destination, then the source starts a new route discovery mechanism[1,6].

\section{AD HOC ON-DEMAND DISTANCE VECTOR(AODV)}

AODV is "on-demand" ora destination based reactive protocol, whichprovides on-demand route discoveryand maintain a route to another node until the two needs to communicate. This protocol inherits the feature of route discovery from DSR. However, AODV resolves the problem of large headers found in DSR. AODV maintains routing tables on the nodes instead of including a header in the data packet. The routing decisions are made using distance vectors, i.e. distances measured in hops to all available routers $[6,19]$.

Route finding in AODV is based on a route discovery cycle involving a broadcast network search and a unicast reply containing discovered paths. Each node maintains a sequence number which saves a time stamp and for loop freedom, 
and a routingtable which contains routes to destinations. Sequence numbers are used to determine the freshness of routes (thehigher the number, the fresher the route which allows the older one to be discarded). Since this is an ondemand distance vector scheme (routersmaintain distances of only those destinations that they need to contact or relay information to). Each active routeis associated with a lifetime stored in the table; if a route is not utilized within the lifetime period, the route is marked as invalid and later on, removed. Otherwise, each time the route is used, the lifetime period is updated so that the route is not prematurely deleted $[8,9]$.

The two main mechanisms used by the AODV protocol to establish and maintain the connection are [6]:

1. Route Discovery mechanism.

2. Route Maintenance mechanism

Four types of control packets are used to discover the path in the network if required. As shown in Figure 2 [Chakeres and Royer] [20].

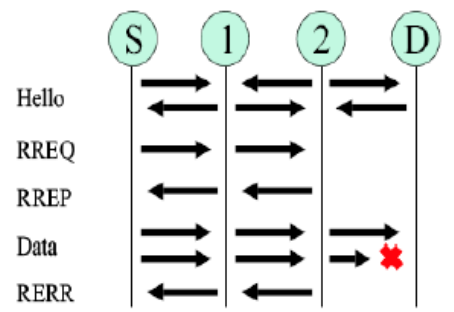

Figure 2: AODV protocol messaging [20]

\section{Route Discovery mechanism in AODV}

Thesource node initiates the route discovery process in the same way as in DSR. An intermediate node may replywith a route reply (RREP) only if it knows a more recentpath than the one known by the sender node to thedestination. A new routerequest generated by the sender node is tagged with ahigher sequence number and an intermediate node thatknows the route to the destination with a smaller sequencenumber cannot send the RREP message. Forward links aresetup when a RREP travels back along the path taken byRREQ. So the routing table entries are used to forward thedata packet and the route is not included in the packetheader[19].

\section{Route Maintenance mechanism in AODV}

In AODV, the role of route maintenance is to provide feedback to the sender in case a link breakage occurs, to allow the route to be modified or re-discovered as follow: If a source node moves, then it must rediscover a new route. If an intermediate node moves, its upstream neighbor notices the move and propagates a link failure route error (RERR) message to each of its active upstream neighbors. These nodes in turn propagate RERR, and so on, until the source node is reached. The source node may re-initiate route discovery if a route is still desired.AODVprotocol uses hello messages , which are periodic local broadcasts made by a node to advertise its presence in its neighborhood.. A node learns that a link is broken when it does not receive a HELLO for a predetermined time[6, 20, 21].

\section{OPTIMIZED LINK STATE ROUTING PROTOCOL (OLSR)}

It is a table-drivenproactive routing protocol. It wasdeveloped for mobile $\mathrm{Ad} \mathrm{Hoc} \mathrm{networks.} \mathrm{So} \mathrm{the} \mathrm{routes} \mathrm{are}$ alwaysimmediately available when needed.OLSR is an optimization of link-state routing;with the message flooding in OLSR is optimized topreserve bandwidth. The optimization is based on atechnique called Multipoint Relaying. Under this protocol, allthe nodes contain pre-computed route information aboutall the other nodes in network. This information isexchanged by protocol messages periodically.Each node selects a set of its neighbor nodes as MultiPoint Relays (MPRs). Only those nodes selected as MPRsare responsible for forwarding the Control Traffic. MPRsare selected such that 2-hop neighbors can be reachedthrough at least one MPR node. MPRs are used to form the route from starting node todestination node in MANET. The purpose of selecting MPRs is to reduceflooding overhead and provide optimal flooding distance as shown in Figure 3 [1, 22, 23].

OLSR uses two kinds of control messages: Hello andTopology Control (TC). Hello messages are used forfinding information about the link status and the host'sneighbors. With the Hello message, the Multipoint Relay(MPR) Selector set is constructed which describes which neighbor has chosen this host to act as MPR. The Hello messages are sent only one hop away but the TC messages are broadcast throughout the entire network. The TC messages are broadcast periodically and only the MPR hosts can forward the TC messages[6]. 


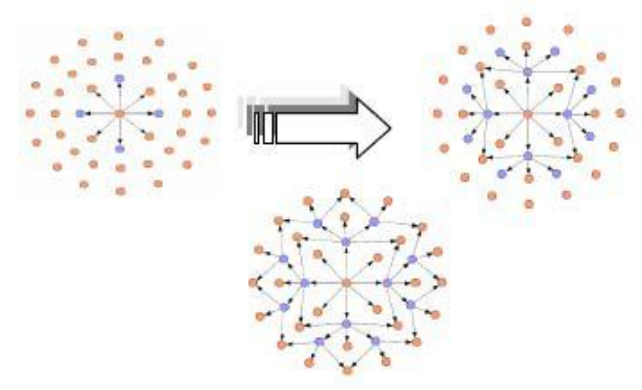

Figure 3: OLSR mechanism [22]

\section{VII.RANDOM WAYPOINT MOBILITY MODEL}

The Random Waypoint (RWP) mobility model is the simplest and most widely available model for MANETS studies [1]. The algorithm for RWP is as follows:

1. A node chooses a random destination anywhere in the network area.

2. The node starts moving towards the destination witha velocity randomly chosen from a speed vector[Vmin,Vmax].

3. After reaching the destination, the node stops at the destination for a duration specified by 'pause time' parameter, which is the same for all nodes.

4. All nodes repeat this procedure until the simulationends.

\section{PROTOCOLS PERFORMANCEMETRICS}

The MANET working group within the International Engineering Task Force(IETF) has defined some unique characteristics of Ad Hoc networks for standardization in Internet Drafts or Request for Comments(RFC 2501)(request for comment) [24], which describe very nature of Ad Hoc networks. The following four quantitative [5, 19] performance metrics are used for this study:

1. Network Throughput:It gives the fraction of the channel capacity used for useful transmission and is defined as the total number of packets received by the destination. It is in fact a measure of the effectiveness of a routing protocol.

2. Average packettransmission time delay:This includes all possible delays caused by buffering during route discovery latency, queuing at the interface queue, retransmission delays at the MAC, and propagation and transfer times.

3. Routing overhead:It is the total number of routing packets transmitted during the simulation.. All the packets sent or forwarded at network layer are considered as routing overhead.

4. Packettransmissionratio (PTR): The ratio of the data packets delivered to the destinations to those generated by the traffic sources.

All these metrics are most widely used for representing

performance of routing protocols because higher data delivery, lower control overhead and lower delay are always desirable. The essential parameters, which we have varied in our simulations, are mobility speed and pause time and network size [5].

\section{SIMULATION SCENARIO AND PARAMETERS SETUP}

OMNeT++ version 4.1Simulator was used to model MANET scenarios as shown in Figure 4.The model was designed with an extensive set of parameters and was used to evaluate, analyze, and compare the performance of three routing protocols (AODV, DSR, and OLSR).

In this paper the studied scenarios have been done on network size of 50 and 100 nodes; node are distributed randomly in a grid network topology and mobile nodes moving with velocities ranges from $1 \mathrm{~m} / \mathrm{s}$ to $30 \mathrm{~m} / \mathrm{s}$ using Random Waypoint mobility models with two values of pause time (0-3) and (200-300) seconds. 


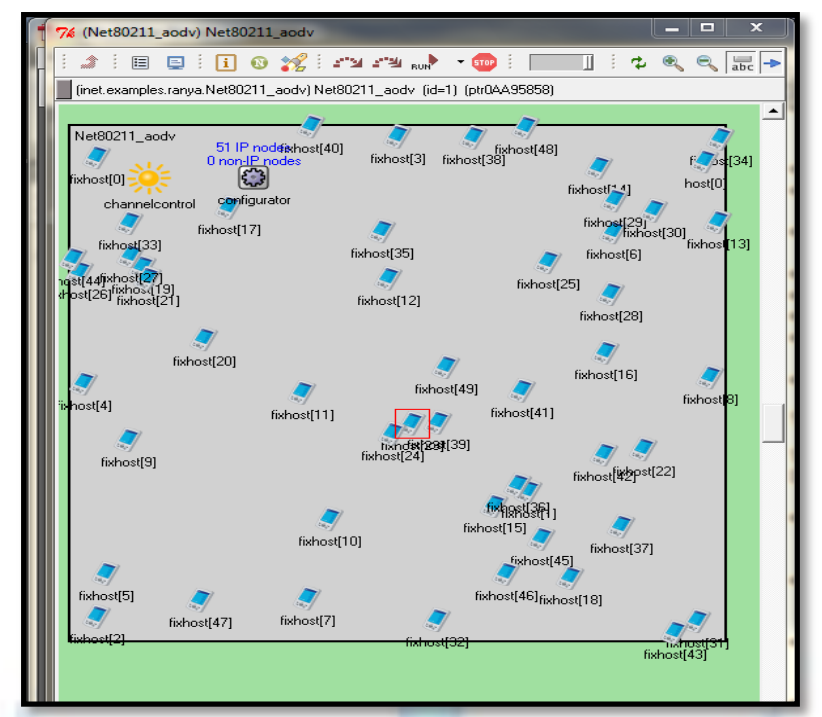

Figure 4: MANET Scenario

Thesimulation of all scenarios has been done for 500 seconds in a simulation area of $500 \mathrm{mX500m}$ with CBR (UDP) traffic pattern. The source-destination pairs are spread randomly over the network. The size of data packets is 1024 bytes withpacket rate of 10 packets per second. Simulations are carried out by network load with ten source nodes send data to ten destination nodes at the execution time. To overcome the effect of randomness in the output we have taken the averages of the results to get their realistic values.

The simulation parameters are shown in Table 1.

Table 1: Parameters Setup

\begin{tabular}{|l|l|}
\hline \multicolumn{1}{|c|}{ Parameter name } & value \\
\hline Size of playground & $500 \mathrm{~m}^{*} 500 \mathrm{~m}$ \\
\hline Number of fix hosts & 50,100 \\
\hline No. of mobile hosts & 10 \\
\hline Packet size & $1024 \mathrm{~B}$ \\
\hline Packet rate & 10 packet/s \\
\hline Simulation time & $500 \mathrm{~s}$ \\
\hline Data Traffic pattern & CBR,UDB \\
\hline Node speed & $1,3,8,10,15,20 \mathrm{and} 25 \mathrm{~m} / \mathrm{s}$ \\
\hline Mobility model & random waypoint \\
\hline Radio bit rate & $54 \mathrm{Mbps,2.4MHZ}$ \\
\hline MAC Layer & leee.802.11g wifi \\
\hline Pause Time & $(0-3)$ and $(200-300) \mathrm{s}$ \\
\hline
\end{tabular}

\section{EXPERIMENTAL RESULTS AND PERFORMANCE COMPARISION DISCUSSION}

Two tests were carried out to evaluate andanalyze the impact of node speed variationson theperformance of AODV,DSR and OLSR at using 50 and 100 network nodes density for grid network structure. In order to show the effect of pause time variation on the performance of routing protocols, for each network size, two experiments were runfor two values of pause time (0-3) and (200-300) seconds.(0 -3) s means continuously moving (high mobility)and (200-300) means stable or low mobility. When the nodes are continuously moving ( $0 \mathrm{~s}$ pause time) the number of link changes is very high and decreases with increase in pause time and converge to 0 (200s pause time). At this stage the network becomes stable. According to the performance metrics that have been explained in section VIII, the experimental results and performance evaluation of the three protocols are as follow: 
Figure 5 shows that when network size is 50 nodes, DSR is able to achieve slightly more throughput than AODV in less stressful situations (low speeds and long pause time). This could indicate that local repair can be used more efficiently to recover from loss at low speed due to the use of route caching in DSR.While when node speed increases in stressful situations (i.e., larger number of nodes and higher mobility), the AODV protocol slightly outperforms the DSR protocol with respect to the network throughput as shown in Figure 9 for network size of 100 nodes. This is because the AODV protocol works better in stressful situation with a heavy network load and high mobility, while DSR work better in network with low density network. However, OLSR gives comparatively lower throughput as the large number of routing bits is required for keeping update network topology and fresh routes; increase in overhead reduces the throughput which will in turn have effect on the delivery ratio of packets due to the fact that when topology changes more quickly protocol control messages will increase more which consumes more bandwidth that affects network throughput and packet delivery ratio as shown in Figure 6.

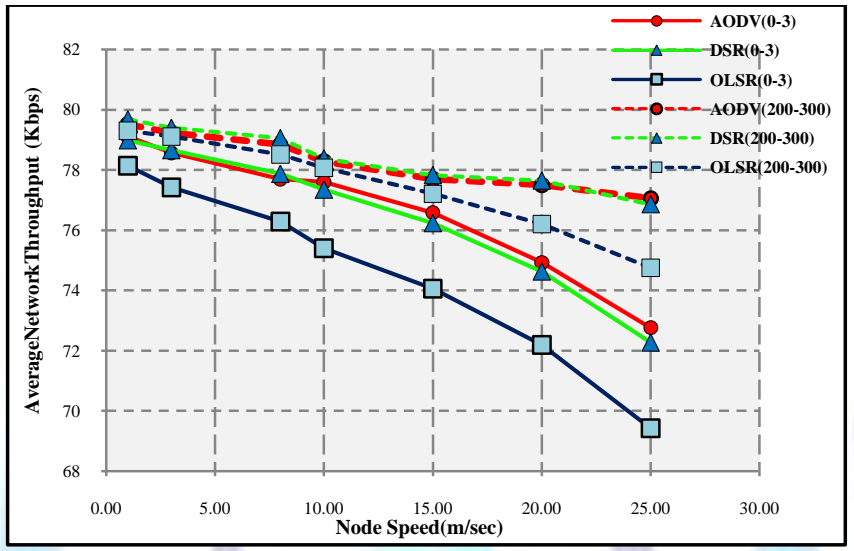

Figure 5: Average Network Throughput (Kbps) vs. node speed $(\mathrm{m} / \mathrm{s})$ for 50 nodes

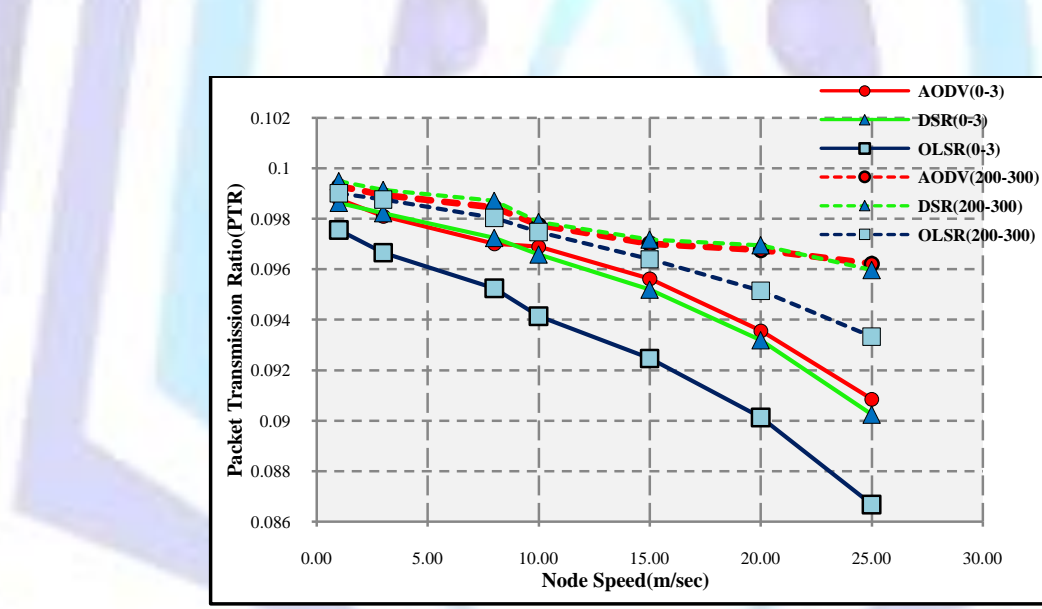

Figure 6: packet transmission ratio (PTR) vs. node speed $(\mathrm{m} / \mathrm{s})$ for 50 nodes

Figure 7 shows that the average packet transmission time delays versus node speed for all three routing protocols for different pause time values. The packet transmission time delay increases with an increase in node speed for all three protocols. In reactive protocols, if there is no route to a destination, packets to that destination will be stored in a buffer while a route discovery procedure is conducted which takes some time. Moreover, OLSR continuously maintains routes to all destinations in the network. When link break occurs, it can quickly find a new route to the destination since the routing table has routes for all available hosts in the network. Figure7 also shows thatthe packet transmission time delay of DSR is the lowest as compared to AODV. This is likely because the route acquisition procedure in DSR allows more routes to be detected and cached than in AODV, which obtains a single route per RREQ. With a higher mobility and high traffic load, links are more frequently broken, for DSR, packets wait less time during route acquisition than with AODV. In OLSR, routes are known well in advance; hence the average packet transmission delay is less than that of AODV and DSR. 


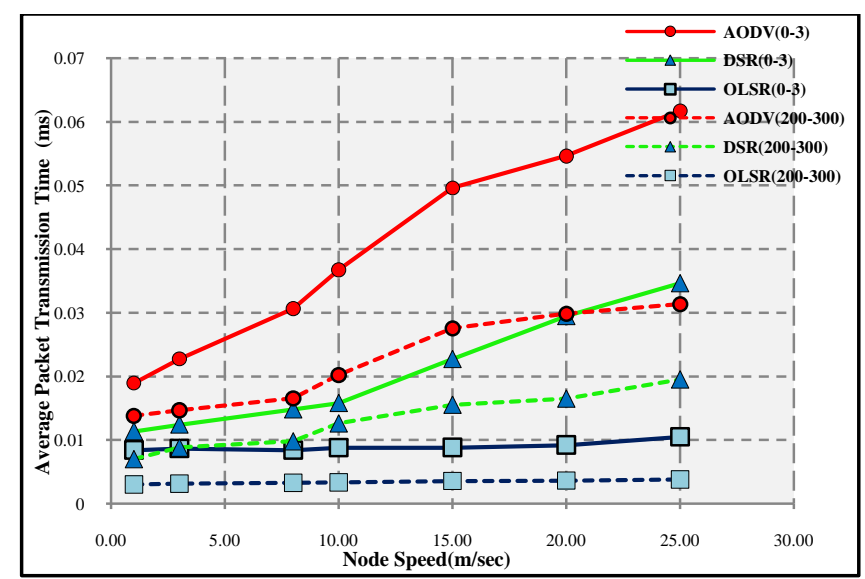

Figure 7:Average Packet Transmission Time vs. node speed $(\mathrm{m} / \mathrm{s})$ for 50 nodes

Protocol overhead represents total number of bytes generated by a routing protocol for routing operationswithin a network. Increases in protocol overhead will negatively affect the network performance by consumingbandwidth. Figure 8 shows that DSR creates the least overhead as compared to both AODV and OLSRat high mobility (short pause time), this is due to the way routes are detected in DSR; also the route acquisition procedure in DSR allows more routes to be detected and cached with the same RREQ than in AODV (which obtains a single route per RREQ), and this reduces the protocol overhead in DSR.OLSR shows a higher difference as compared to the other two protocols.Because OLSR is atable-driven protocol, and the proactivity nature of OLSR makes it exchange topology information with other nodes of the network regularly and periodically, this increases the overhead. This figure demonstrates also that with high mobility, DSR overhead is increasing more, this is because of source routing mechanism of DSR.

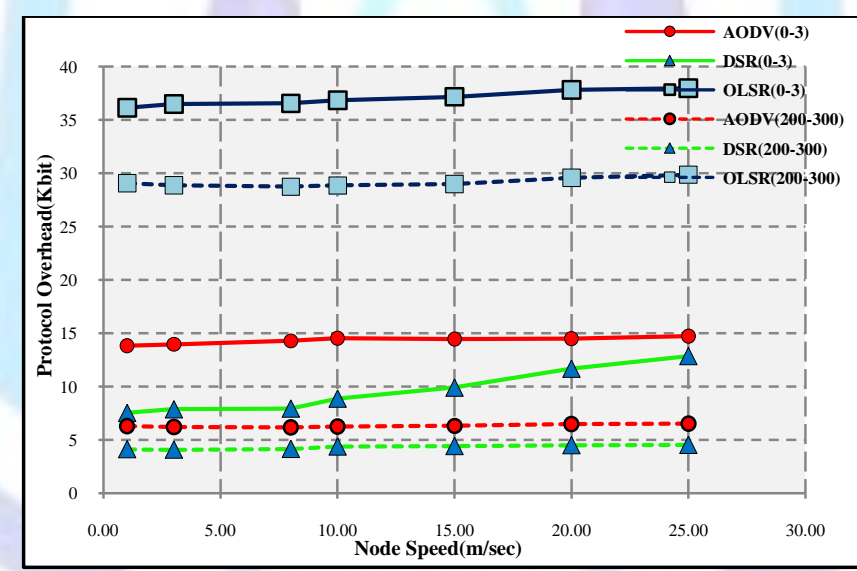

Figure 8: Protocol overhead vs. node speed $(\mathrm{m} / \mathrm{s})$ for 50 nodes

Figure 9 demonstrates that AODV outperforms the other two protocols (DSR and OLSR) for all pause time values considered.Also this figure showsthat the performance of DSR is significantly poorer as node density increases. DSR exhibits severe degradation in performance with higher node density. This is an effect of the higher network load caused by the source routes carried in all data packets, therefore, at higher mobility and when using a heavy network load, AODV appears to be a better protocol choice than DSR for MANET applications. However, the OLSR performance is less than the performance of both DSR and AODV in dense and unstable network (short pause time). This is because a large amount of overheads generated to keep network updated, this overhead consumes the network bandwidth, consequently degrades the network performance. This figure demonstrates also that the throughput's decrease percentage is slight at about $1.8 \%$ when using the AODV routing protocol, and less than the throughput's percentage decrease when using other protocols (DSR, by about $1.9 \%$ and OLSR, by about $2.4 \%$ ). 


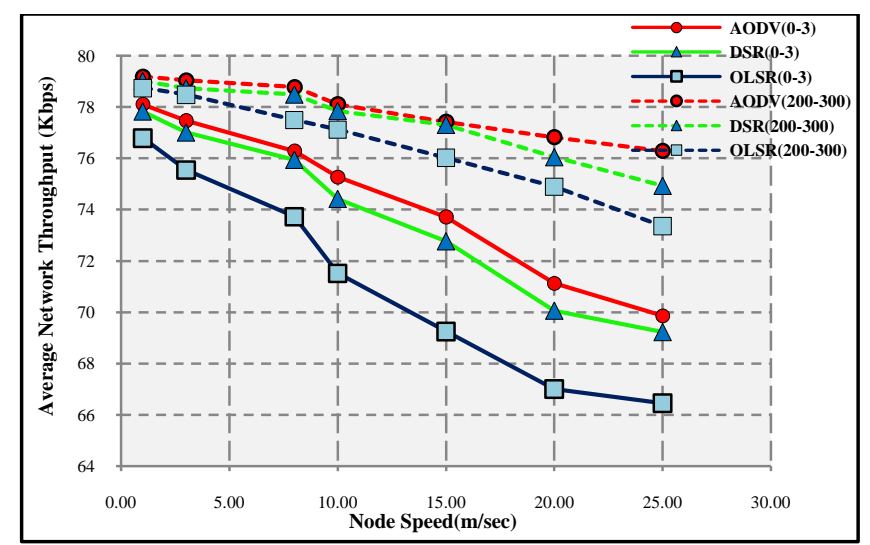

Figure 9: Average Network Throughput (Kbps) vs. node speed (m/s) for 100 nodes

Packet Transmission Ratio (PTR) describes the loss rate that will be seen by the transport protocols, which in turn have affect the maximum throughput that the network can support. For each protocol, the network PTR starts to decrease with an increase in node speed. The PTR shown in Figure 10 reveals that AODV shows better performance than DSR in both cases (short and long pause times) because of the effect of the higher network load caused by the source routes for DSR carried in all data packets. Therefore, at higher mobility (short pause time), packet loss due to an increase in speed of the mobile nodes decreases the packet transmission ratio (PTR) which leads to make AODV and OLSR more robust than DSR

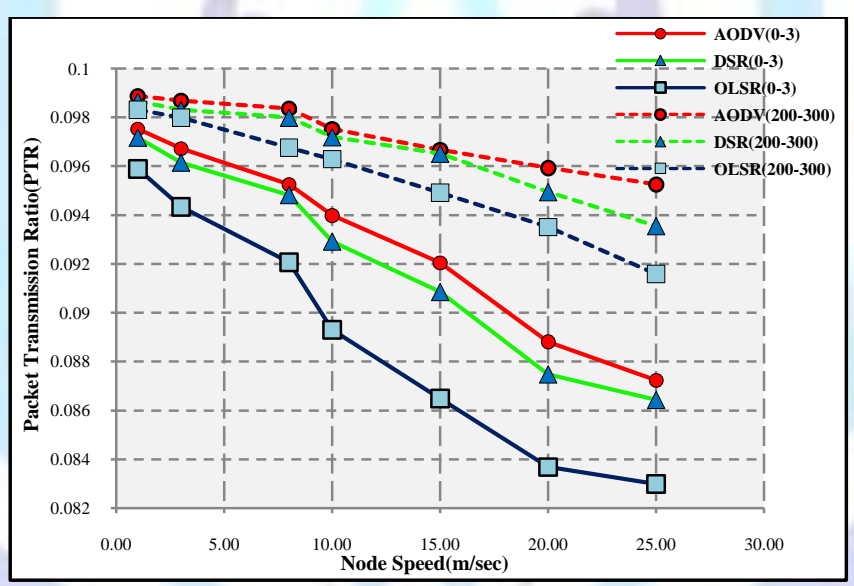

Figure 10: packet transmission ratio (PTR) vs. node speed $(\mathrm{m} / \mathrm{s})$ for 100 nodes

Figure 11, shows a strong rise in packet transmission time delay of DSR as the number of nodes increases from 50 to 100. This may be due to large overhead packets in DSR as compared with AODV. Higher node density increases the number of neighboring nodes and that causes more route reply messages to the source node with large packet header used in DSR, and also stale routes are held for a long time thus increasing time delay. AODV shows the lower delay than DSR for high node speed. In AODV better technique is used, as the destination replies only to the first arriving RREQ. This automatically favors the least congested route instead of the shortest route. Whilst, DSR replies to all RREQs, which makes it difficult to determine the least congestion route. OLSR reacts well to the link breakage because OLSR continuously maintains routes to all destinations in the network and when link break happens, it can find a new route, there is no data buffering for invalid routes to the destination faster than DSR. With the higher node density, overall transmission time delay for all three protocols increases as the number of hopes increases. In these cases the packet needs to cover long distance to reach the destination. AODV performs better in stressful situation (high load or high mobility). 


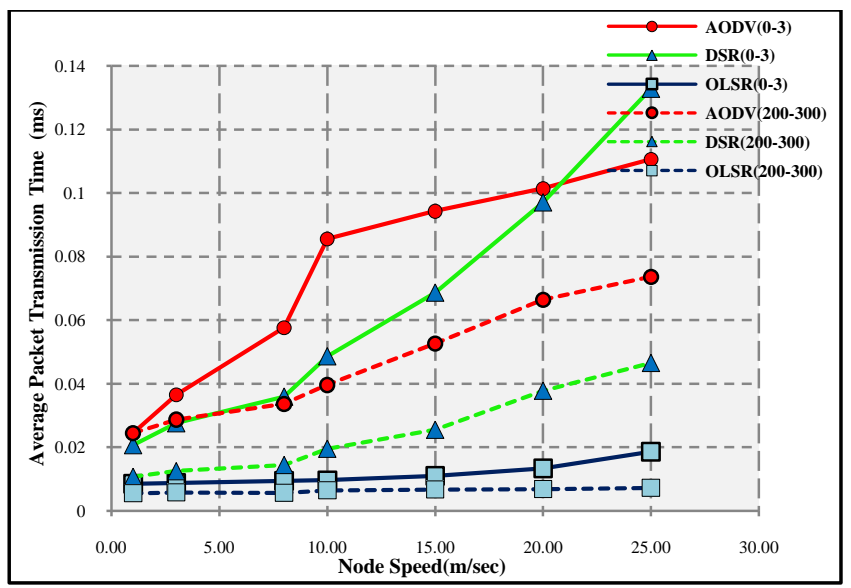

Figure 11:Average Packet Transmission Time vs. node speed $(\mathrm{m} / \mathrm{s})$ for 100 nodes

Figure 12 shows the protocol overhead versus node speed for different pause times. It demonstrates that the DSR overhead is lower than OLSR and AODV overheads for both pause time values in case of low speed. When there is an increase in the speed of the node, the OLSR overhead still remains higher than DSR and AODV, likely due to the proactive nature of OLSR. On the other hand, DSR overhead is higher than the AODV overhead, because DSR is based on source routing algorithm and every data packet must hold the entire route from the source to the destination in its header. In this stage poor performance with using DSR may be explained by the aggressive route caching technique built in this protocol, but for a higher node density, the benefit of caching routes seems to be lost.

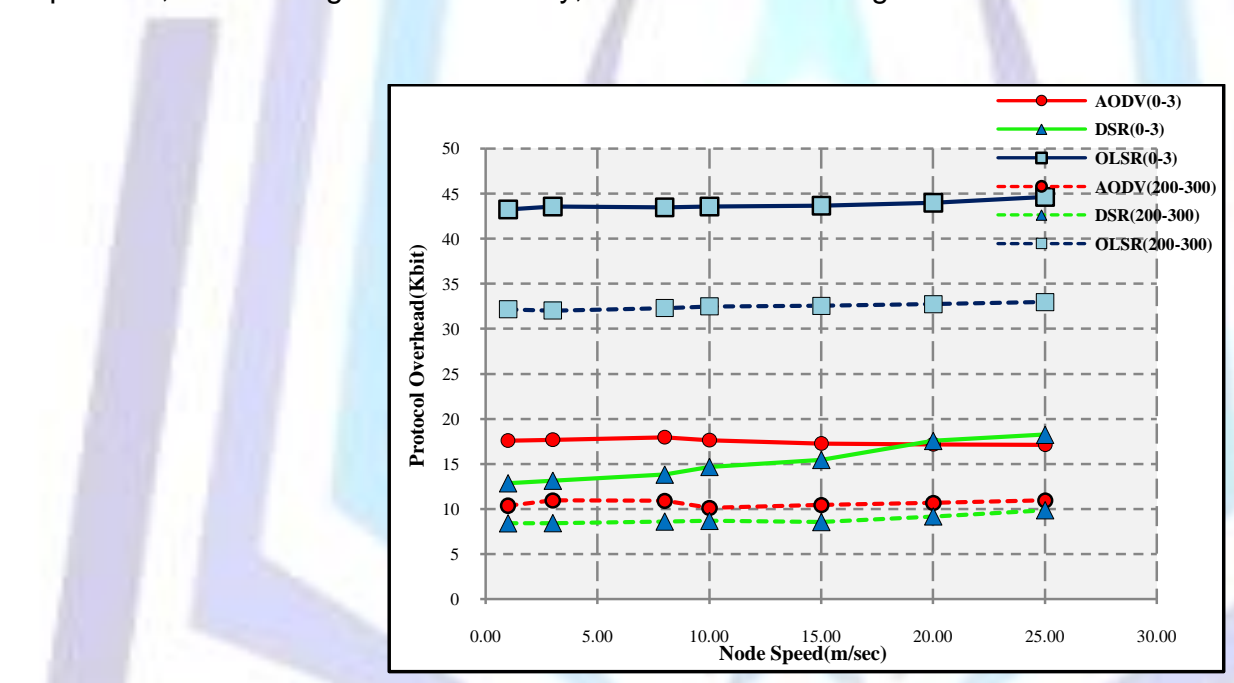

Figure 12: Protocol overhead vs. node speed $(\mathrm{m} / \mathrm{s})$ for 100 nodes

\section{CONCLUSION}

In this paper, the simulation results show some important differences among the performance of MANETrouting protocols in terms of network throughput, packet delivery ratio, routing overhead and packet transmission time delay. The presence of high mobility implies frequent link failures and each routing protocol reacts differently during link failure.

Under varying speeds and with a varying network size, AODV outperforms DSR with respect to network throughput and packet transmission ratio in stressful situations (heavy traffic load and/or high mobility, network size is large). DSR protocol, however, consistently generates less routing overhead than AODV.It has to be noted that for the light network situation, DSR shows better performance. The OLSR is outperformed by both other protocols for all mobility and load test situations.

DSR outperforms AODV with respect to packet transmission time delay for all speed values due to the caching mechanism used by DSR. On the other hand, OLSR provide a better quality of service than both DSR and AODV, this is dueto OLSR proactive naturewhich makes routes to every destination are always available and up-todate resulting minimumtime delay. While For on-demand protocols, DSR and AODV, the source node has to wait for the route to bediscovered before transmission. This delay in route discovery might be unsuitable for real-time applications. However, this delay advantage of OLSR is on the expense of high protocol overhead which consumes some of the available bandwidth and consequently reduces network throughput. 


\section{REFERENCES}

[1] S. K. Sarkar,T. G. Basavaraju and C. Puttamadappa, "Ad Hoc Mobile Wireless Networks", Auerbach Publications Taylor \& Francis Group, 2008.

[2] A. K. Pathan, "Security of Self-Organizing Networks: MANET, WSN, WMN, VANET", 1st Ed., Taylor and Francis Group, USA, 2010.

[3] F. Bai, N. Sadagopan and A. Helmy, " Important: A Framework to Systematically Analyze the Impact of Mobility on Performance of Routing Protocols for Ad Hoc Networks", Twenty-Second Annual Joint Conference of the IEEE Computer and Communications. IEEE Societies, Vol.2, pp. 825 - 835, 30 March- 3 April 2003.

[4] F. Bai, et al., "Modeling Path Duration Distributions in MANETs and Their Impact on Reactive Routing Protocols", IEEE Journal On Selected Areas in Communications, Vol. 22, No. 7,pp.1357-1373, September 2004.

[5] S. Kumar, R. K. Rathy and D. Pandey, "Traffic Pattern Based Performance Comparison of Two Reactive Routing Protocols for Ad Hoc Networks Using NS2", Computer Science and Information Technology, ICCSIT, 2nd IEEE International Conference on, pp. 369-373,8-11 August 2009.

[6] L. A. Hassnawi, et al., " Performance Analysis of Various Routing Protocols for Motorway Surveillance System Cameras' Network", International Journal of Computer Science (IJCSI), Vol. 9, Issue 2, No.1, pp. 369-373, March 2012.

[7] Y. Ho and W. Chang, "The Influence of Link Break on Wireless Ad Hoc Networks", Parallel and Distributed Processing with Applications (ISPA), International Symposium , pp. 175 - 180, 6-9 September 2010.

[8] R. S. Kumar and P. Kamalakkannan, " A Novel Energy Based Routing Algorithm to Reduce Link Break in Mobile Ad Hoc Networks", International Journal of Innovative Technology and Exploring Engineering (IJITEE), Vol.2, Issue 5, pp. 215-220, April 2013.

[9] Preetha, A. Unnikrishnan and K P. Jacob, "A Probabilistic Approach to Reduce the Route Establishment Overhead in AODV Algorithm for MANET", International Journal of Distributed and Parallel Systems (IJDPS) Vol.3, No.2, pp.207-214, March 2012.

[10] B. Divecha, et al., "Impact of Node Mobility on MANET Routing Protocols Models", Tata Institute of Fundamental Research, pp.1-11, 2007.

[11] S. Gowrishankar, et al., "Scenario Based Performance Analysis of AODV and OLSR in Mobile Ad Hoc Networks", International Journal of the Computer, the Internet and Management, Proceedings of the 24th South East Asia Regional Computer Conference, Vol. 5, pp. 8.1-8.6, November 18-19, 2007.

[12] X. Hu, et al., "Is Mobility Always Harmful to Routing Protocol Performance of MANETs?", Cyber-Enabled Distributed Computing and Knowledge Discovery (CyberC) International Conference, pp. 108 - 112, 10-12 Oct. 2010.

[13] M. Amnai, Y. Fakhri and J. Abouchabaka, "Evaluation of Impact of Traffic VBR and Mobility on the Performance of AODV Routing Protocols in Mobile Ad hoc Networks", Multimedia Computing and Systems (ICMCS) International Conference, pp. 1 - 5, 7-9, April 2011.

[14] M. Alshowkan, E. A. Fattah and A. Odeh, "Performance Evaluation of DYMO, AODV and DSR Routing Protocols in MANET", International Journal of Computer Applications, Vol. 49, No.11, pp. 29-33, July 2012.

[15] T. Clausen and P. Jacquet, " Optimized Link State Routing Protocol (OLSR)", Internet Engineering Task Force, RFC: 3626, October 2003.

[16] D. Johnson, Y. Hu and D. Maltz, " The Dynamic Source Routing Protocol (DSR) for Mobile Ad Hoc Networks for IPv4", Internet Engineering Task Force, RFC: 4728, February 2007.

[17] C. Perkins, E. Belding-Royer and S. Das, "Ad Hoc On-Demand Distance Vector (AODV) Routing", Internet Engineering Task Force, RFC: 3561, July 2003.

[18] K. Shanwaz and D. Sharath, "Reducing Link Failures in MANETs Using Link Breakage Prediction Algorithm", International Journal of Engineering Research \& Technology (IJERT), Vol. 1 Issue 6, pp.1-6, August 2012.

[19] K. Amjad and A.J. Stocker, "Impact of Node Density and Mobility on the Performance of AODV and DSR in MANETS", Communication Systems Networks and Digital Signal Processing (CSNDSP), 2010 7th International Symposium, pp. 61 - 65, 1-23 July 2010.

[20] J. Jain, R. Gupta and T.K. Bandhopadhyay, " On Demand Local Link Repair Algorithm for AODV Protocol", International Journal of Computer Applications, Vol. 35, No.5, pp.20-25, December 2011.

[21] E. Ravindra, V. V Kohir and V. D Mytri, "A Local Route Repair Algorithm Based On Link Failure Prediction in Mobile Ad Hoc Network", World Journal of Science and Technology, pp.64-67, 2011. 
[22] P.Jacquet, et al., "Optimized Link State Routing Protocol for Ad Hoc Networks ", Multi Topic Conference IEEE INMIC, pp. $62-68,2001$.

[23] [38] T. Lin, "Mobile Ad-Hoc Network Routing Protocols: Methodologies and Applications", Ph.D. Dissertation Computer, Virginia Polytechnic Institute and State University, 19th March 2004.

[24] S. Corson and J. Macker, "Mobile Ad Hoc Networking (MANET): Routing Protocol Performance Issues and Evaluation Considerations", Internet Engineering Task Force, RFC: 2501, January 1999. 